\title{
Systematic assessment of the technical features of seventeen automated external defibrillators and their potential implication in the clinical setting
}

\author{
Fulvio Kette $^{1 *}$, Aldo Locatelli ${ }^{2}$, Marcella Bozzola ${ }^{2}$, Guido F Villa ${ }^{2}$, Alberto Zoli ${ }^{2}$, Carlo Picco ${ }^{2}$ and Aida Andreassi ${ }^{2}$ \\ ${ }^{1}$ Company for Health Care 5 Friuli Occidentale, Pordenone, Italy \\ ${ }^{2}$ AREU, Azienda Regionale Emergenza Urgenza Lombardia (Lombardy Regional Emergency Service, Milan), Italy
}

\begin{abstract}
Aim: Assessment and comparison of technical features of seventeen Automated External Defibrillators (AEDs).

Method: Engineering bench tests for a systematic descriptive evaluation in commercially available AEDs. The devices were tested through an ECG simulator, an impedance simulator, an oscilloscope and a measuring device. All tests were performed at the engineering facility of the Lombardy Regional Emergency Service (AREU).

Results: The AEDs highlighted large variations in terms of physical features, time intervals from lighting to the ready-for-analysis time interval, time required to analyse shockable and non-shockable rhythms, voice prompts and other additional features such as a metronome and feedback devices aimed to obtain the best quality of chest compression (depth and rate).

As per the size, they highlighted similar characteristics in term of weight and size but one that resulted substantially smaller and lighter. Their pre-set energy was found to range between 150 and 360J. The lighting time varied from a minimum of $9.5 \mathrm{sec}$ (Nihon Kohden Cardiolife) to $44 \mathrm{sec}$ (Ami Italia Saver One). The time spent for the analysis was predominantly shorter in ventricular fibrillation (VF) than in non-defibrillating rhythms. A metronome was present in 11/17 devices whereas an accelerometer was present in two AEDs only. The voice prompts also differed ranging from "begin CPR" to diverse types of information including compression-toventilation ratio, elapsed time and the effectiveness of cardiac massage.

Conclusions: There is a large variability in the physical characteristics of the AEDs tested. Some feature may not affect the quality of the devices, such as the size or the type of waveform whereas others (i.e. time for lighting, time spent for the analysis) may hamper the effectiveness of successful resuscitation due to the long time required without performing chest compression. Accordingly, some peculiarity should be improved to better fulfil the requirements of the current Guidelines for Cardiac Arrest and CPR, particularly to minimize the interruption of chest compression.
\end{abstract}

\section{Introduction}

Automated external defibrillators (AEDs) have become increasingly available through a wide spread diffusion $[1,2]$. They early use by lay people together with prompt onset of chest compression is associated with increased successful outcome up to 50 to $70 \%$ [3-7]. Whenever a person collapses with high suspicion of cardiac arrest and an AED is not immediately available, chest compression (CC) should be initiated as quickly as possible to maintain the heart more susceptible to a subsequent defibrillation in case of shockable rhythms. Thus, during resuscitation maneuvers, CPR and defibrillation are tightly linked.

When manual defibrillators are used, chest compression is interrupted only when the operator is ready to provide the shock after which CC must be restored immediately [8]. When AEDs are deployed, however, the operators must respect the indications of the devices by not touching the patient until the rhythm analysis is completed, and the capacitor is fully charged. During this time interval, chest compression is interrupted in order not to interfere on the rhythm analysis. This situation may critically reduce the hands-on time, in contrast to the evidence that the shortest is the delay from CC to the shock (and vice versa) the better is the likelihood of a successful restoration of spontaneous cardiac function [9-12]. Accordingly, current guidelines state that "AEDs make it possible to defibrillate many minutes before professional help arrives" and "pre-shock and post-shock pauses in chest compressions should be as short as possible" [13]. In the setting of a thorough investigation on several AEDs we analysed their behaviour under the electrical point of view and on ventricular tachycardia [1416]. We identified several differences in current delivered, in the energy provided by the devices, in the waveforms as well as in the recognition of defibrillating rhythms. It is in this context that we observed different features on the AEDs examined. We therefore decided to make additional systematic measurements on other technical features in 17 commercially available AEDs.

${ }^{\star}$ Correspondence to: Fulvio Kette, Company for Health Care 5 Friuli Occidentale, Pordenone, Italy, Tel: +39 0434369 975; E-mail: fulvio.kette@aas5.sanita.fvg.it

Key words: Automated External Defibrillators, AED, rhythm analysis, ventricular fibrillation

Received: August 31, 2018; Accepted: September 17, 2018; Published: September 20, 2018 


\section{Methods} tested.

Overall, seventeen AEDs from twelve different manufactures were

- Saver One (Ami Italia, Napoli, Italy);

- G3 Pro (Cardiac Science, Bohtell, Washington, USA);

- Lifeline AED (Defibtech, Guilford, Connecticut, USA);

- Responder AED (General Electric, Schenectady New York, USA);

- Sam300P (Heart Sine, Belfast, Ireland);

- Lifepak 1000 (Physio Control, Redmond, Washington, USA);

- Lifepak Express (Physio Control, Redmond, Washington, USA);

- Cardiolife 2100 (Nihon Kohden, Shangai, China);

- FR2+ (Philips, Eindhoven, Netherlands);

- FRx (Philips, Eindhoven, Netherlands);

- FR3 (Philips, Eindhoven, Netherlands);

- Rescue SAM (Progetti, Trofarello, Italy);

- AED Heart Save (Primedic, Rottweil, Germany);

- FRED Easy (Schiller, Baar, Switzerland);

- FRED Easyport (Schiller, Baar, Switzerland);

- AED Plus (Zoll, Chelmsford, UK);

- AED Pro (Zoll, Chelmsford, UK).

The following characteristics were analyzed:

- Size and weight

- Time of lighting

- Time of analysis in defibrillating and non-defibrillating rhythms. As shockable rhythms we considered coarse ventricular fibrillation (VF). Data on ventricular tachycardia are reported elsewhere [15]. For non-shockable rhythms we evaluated: sinus rhythm, atrial fibrillation, junctional, asystole, $3^{\text {rd }}$ degree AV block, idioventricular rhythm. For each rhythm the time intervals from the vocal messages "Don't touch patient. Analyzing" to "Shock indicated" or "Shock not indicated" were measured.

- Vocal prompts. Due to the differences in each device, the audio information provided by the AEDs were summarized into four categories:

1. Message "begin CPR";

2. Message "begin CPR" plus the compression to ventilation ratio prompt;

3. Message begin CPR" plus information regarding the elapsed time;

4. Message "begin CPR" plus information regarding the effectiveness of cardiac massage.

- Presence of CC-assisting devices such as metronome and/or accelerometer.

Tests were made at the Engineering Facility of the Lombardy Regional Emergency Service (AREU, Regional Emergency Urgency Company). In order to avoid bias measurements, all test was made by a single engineer who consistently performed all evaluations.
Following an update of the software, in January 2018 the tests were repeated for the models Saver One (Ami Italia) and Samaritan PAD (Heartsine). These tests were also performed by the same biomedical electronic engineer who performed the earlier assessment.

All tests were repeated three times and the average results were considered.

\section{Results}

Overall, the physical characteristics of the AEDs examined were similar in terms of size and weight. Three models use a rectilinear biphasic waveform (RLBW) (Heartstart Primedic, AED Plus and AED Pro from Zoll), two models adopt a pulsate truncated exponential waveform (Fred Easy and Fred Easy Port from Schiller) whereas all remaining devices adopt the truncated exponential biphasic waveform (TEBW). There are, however, substantial differences in the pre-set energy as nine AEDs are set on 150J, five at 200J, one at 360J while two RLBW are set at 120J. The detailed information is summarized in Table 1. The time window of the phases of lighting, starting analysis, charging the capacitor and delivering the shock (when required) is depicted in Figure 1. The time of lighting substantially differed among the models. From a minimum of 9.5 seconds (Cardiolife, Nihon Kohden) it rose up to 44 seconds (Saver One, Ami Italia). From an average level of 23.7 seconds, six devices had a lighting time greater than the mean value whereas in the other devices it ranged between 9.5 and 21 seconds (Figure 2). The time spent for the analysis varied substantially among the AEDs and also within each device when defibrillating or nondefibrillating rhythms were selected. The time to recognize VF varied from a minimum of 4 seconds to a maximum of 16 seconds. In nondefibrillating rhythms one device (FRX, Philips) required a shorter time than in VF recognition, four had the same as in VF and five required a longer recognition time than in ventricular fibrillation (Table 2).

The message prompts provided by every AED were markedly different. The vocal prompts varied from simple and short messages to prolonged information lasting up to 30 seconds.

A metronome was present in 11 of the 17 AEDs tested whereas a CPR quality feedback device was present in two devices only (AED Plus and AED Pro, Zoll) (Table 3).

\section{Discussion}

Since the introduction of the AEDs into clinical setting, there has been a progressive wide spread diffusion of these devices. Their early use by lay rescuers has been associated with a greater proportion of people successfully defibrillated who were eventually discharged from hospital with intact neurological conditions [3-6]. Since the 2005 Guidelines on Emergency Cardiac Care and Cardiopulmonary Resuscitation the great emphasis given to the defibrillation thus far was overwhelmed by the recognition that the quality of cardiac compression was not as high as generally believed. Previous studies on CPR in animals first and subsequently in human demonstrated that the defibrillation is more effective when the time interval between the shock and CPR is as short as possible $[9-11,17,18]$. Accordingly, a progressive emphasis on the quality of chest compression emerged and efforts were made to improve the quality of chest compression by increasing the compression rate, the depth and the compression fraction to optimize myocardial perfusion $[12,19,20]$.

Differences among AEDs were already recognized by previous independent studies from Achleitner, Snyder, and Rhee's groups [2124]. In addition, the more recent studies by our own research team 
Kette F (2018) Systematic assessment of the technical features of seventeen automated external defibrillators and their potential implication in the clinical setting

Table 1. Characteristics of the AEDs. Kg = kilograms, $\mathrm{cm}=$ centimeters, TEBW $=$ Truncated Exponential Waveform, RLBW = Recti Linear Waveform, $\mathrm{PTE}=$ Pulsate Exponential Waveform, $\mathrm{J}=\mathrm{J}$ Joules

\begin{tabular}{|c|c|c|c|c|}
\hline Model & Weight (Kg) & Size & Wave & Pre-set energy $(\mathrm{J})$ \\
\hline Ami Italia Saver One & 2 & $7,5 \times 21,5 \times 26,5$ & $\mathrm{TE}$ & 150 \\
\hline Cardiac Science G3 Plus & 3,2 & $8 \times 27 \times 31$ & $\mathrm{TE}$ & 200 \\
\hline Defibtech Lifeline & 2 & $7 \times 22 \times 30$ & TE & 150 \\
\hline GE Responder & 3,1 & $8 \times 27 \times 31$ & $\mathrm{TE}$ & 200 \\
\hline Heart Sine Sam 300P & 1,1 & $4,8 \times 20 \times 18,4$ & TE & 150 \\
\hline Nihon Kohden Cardiolife & 2,5 & $10 \times 28 \times 31,5$ & $\mathrm{TE}$ & 150 \\
\hline Philips FR2+ & 2,1 & $6,6 \times 21,8 \times 21,8$ & $\mathrm{TE}$ & 150 \\
\hline Philips FRx & 1,6 & $6,6 \times 22 \times 18$ & $\mathrm{TE}$ & 150 \\
\hline Philips FR3 & 1,6 & $6,1 \times 13,5 \times 23,1$ & TE & 150 \\
\hline Physio Control Lifepak 1000 & 3,2 & $8,7 \times 23,4 \times 27,7$ & $\mathrm{TE}$ & 200 \\
\hline Physio Control Lifepak Express & 2 & $10,7 \times 20,3 \times 24,1$ & $\mathrm{TE}$ & 200 \\
\hline Primedic Heart Save & 2,5 & $9 \times 25 \times 28$ & RL & 360 \\
\hline Progetti Rescue Sam & 2,2 & $9 \times 28 \times 29$ & $\mathrm{TE}$ & 200 \\
\hline Schiller Fred Easy & 1,5 & $7 \times 22 \times 23$ & PTE & 150 \\
\hline Schiller Fred Easyport & 0,49 & $3,5 \times 12,6 \times 13,3$ & PTE & 150 \\
\hline Zoll AED Plus & 3,1 & $13,3 \times 24,1 \times 29,2$ & RL & 120 \\
\hline Zoll AED Pro & 3,12 & $7,6 \times 23,5 \times 23,9$ & $\mathrm{RL}$ & 120 \\
\hline
\end{tabular}

Table 2. Time of analysis (in seconds) for defibrillating and non-defibrillating rhythms. sec $=$ seconds

\begin{tabular}{|c|c|c|}
\hline Device & VF analysis time (sec) & Analysis time of non-shockable rhythms (sec) \\
\hline Ami Italia Saver One & 4 & 19 \\
\hline Cardiac Science G3 Plus & 14 & 14 \\
\hline Defibtech Lifeline & 13 & 12 \\
\hline GE Responder & 8 & 14 \\
\hline Heart Sine Sam300P & 10 & 15 \\
\hline Nihon Kohden Cardiolife & 8 & \\
\hline Philips FRx & 6 & 11 \\
\hline Physio Control Express & 16 & \\
\hline Primedic Heart Save & 9 & 18 \\
\hline Schiller Fred easy & 10 \\
\hline Zoll AED Plus & 10 \\
\hline
\end{tabular}

Table 3. Type of message code (see method section) and presence of metronome or accelerometer

\begin{tabular}{|c|c|c|c|}
\hline Device & Message code & Metronome & Accelerometer \\
\hline Ami Italia SaverOne & 2 & YES & NO \\
\hline Cardiac Science G3 Plus & 2 & YES & NO \\
\hline Defibtech Lifeline & 3 & NO & NO \\
\hline GE Responder & 2 & NO & NO \\
\hline HeartSine Sam300P & 1 & YES & NO \\
\hline Nihon Kohden Cardiolife & 1 & YES & NO \\
\hline Philips FR2+ & 1 & NO & NO \\
\hline Philips FRx & 2 & YES & NO \\
\hline Philips FR3 & 1 & YES & NO \\
\hline Physio Control Lifepak 1000 & 1 & NO & NO \\
\hline Physio Control Lifepak Express & 1 & NO & NO \\
\hline Primedic Heart Save & 2 & YES & NO \\
\hline Progetti Rescue Sam & 1 & YES & NO \\
\hline Schiller Fred Easy & 2 & YES & NO \\
\hline Schiller Fred Easyport & 2 & NO & NO \\
\hline Zoll AED Plus & 4 & YES & YES \\
\hline Zoll AED Pro & 4 & YES & YES \\
\hline
\end{tabular}

further analysed other characteristics thus providing a thorough examination on other features not previously considered. We indeed identified marked variability in the energy, current, first and second phase duration among the AEDs tested as well as in their behaviour when ventricular tachycardia is the underlying rhythm $[15,16]$. In this part of the study we aimed to emphasize other features that distinguish the devices thus completing the set of data acquired during the thorough analysis of their characteristics.

Overall, their sizes are relatively similar except for the model FredEasy Port (Schiller) which is substantially smaller and lighter. Their shapes, however, are different as they were built more likely 


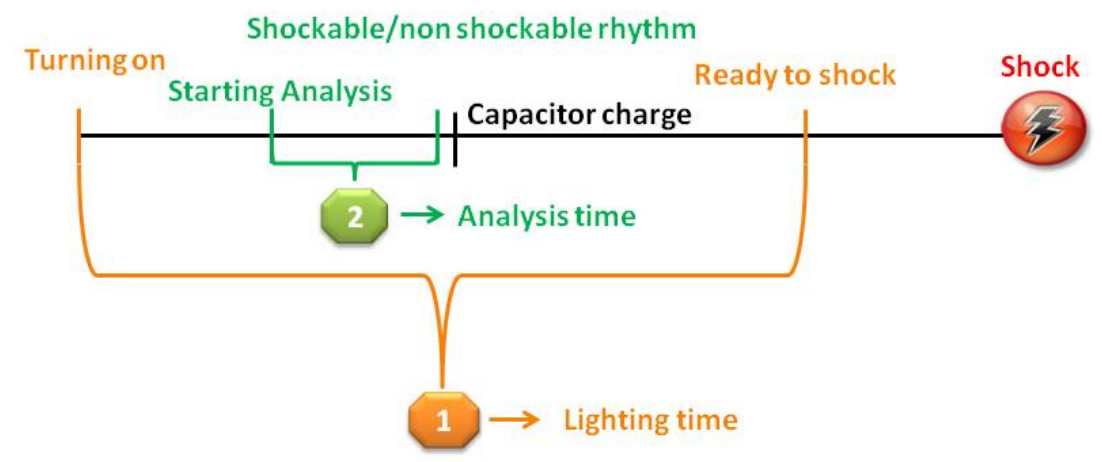

Figure 1. Time windows of the different intervals

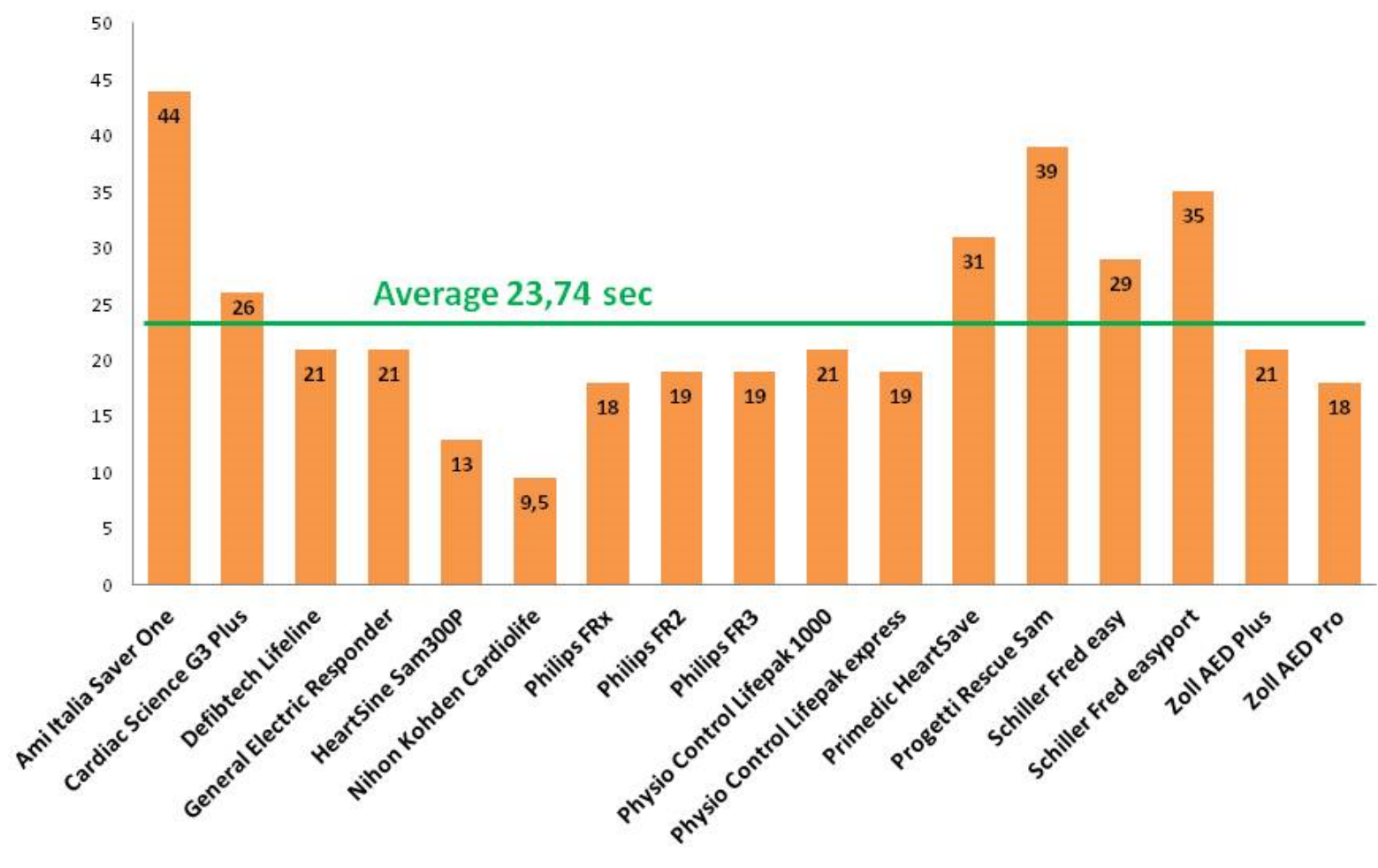

Figure 2. Lighting Time for each AED

to enhance the ergonomy and handling. Although the guidelines recommend an initial energy level of 120J for RLBW and $150 \mathrm{~J}$ for TEBW, we found five devices that were set at $200 \mathrm{~J}$ and one at $360 \mathrm{~J}$ [13]. The manufacturers referred this feature as purposely chosen to enhance the likelihood of converting a VF rhythm since the first shock and to reduce the cumulative energy delivered to the heart.

When we measured the lighting time, we surprisingly noticed that there was an overall long duration lasting up to more than 40 seconds, depending on the models, from the lighting to the moment in which the AED is ready to deliver the shock or, conversely, to suggest to (re) start chest compression in case of non-defibrillating rhythms. During this interval the hands must be kept off the thorax to allow all sequential steps required by the devices. This situation can really endanger the likelihood of converting a VF rhythm due to a prolonged time-period without chest compression.

Similarly, when we measured the time required to analyze a shockable or non-shockable rhythm once the AEDs were already turned on, there was an average time of 10 seconds among the AEDs ranging from a minimum of 4 seconds up to 16 seconds. This time interval was even longer in most AEDs when non-defibrillating rhythms were selected. In both circumstances, too many seconds without chest compression would hamper the success of converting a VF or restoring a viable rhythm.

Comparable results were found when Snyder and co-workers in their investigation on seven AEDs measured the hands-off time from the AED's command to stop compression and the command to deliver the shock. A wide range of time intervals was found from approximately 5 seconds to as high as 28 seconds. ${ }^{23}$ A subsequent study by Berg and collaborators in their retrospective analysis on traces from AEDs used in adult VF patients highlighted a median time of 38 seconds between the first shock and the initiation of chest compression half of which delay was attributed to mechanical/electronic factors of the devices whereas the remaining were related to human factors [25]. 
Similar findings were more recently published by Savastano and co-workers who tested the performances of 12 commercially available AEDs both in manikins and in the real scenario [26]. In their study they confirmed that the hands-off intervals are related to the prolonged times required by the devices to perform the analysis necessary to identify the underlying rhythms either indicating a shock or suggesting chest compression.

The last features we analyzed were related to the prompts and the CPR assisted devices although not tested in all AEDs. We found different types of prompts which were grouped into four categories. This exemplification, however, did not provide the specific characteristics of each device as they were quite different from one model to another. After having listened to the several voice prompts, we reached a conclusion that too few voice information could be insufficient whereas too many could somehow interfere rather than help the operator by producing some sort of "noisy disturbance" in the rescue environment. In addition, a potential delay in restoring the rescue maneuvers might occur when an operator must pay attention to all vocal information provided by the instrument. Indeed, Rhee and co-workers identified better performances by both expert rescuers and lay people by eliminating unnecessary and misunderstanding voice prompts which, together with a capacitor pre-charge, lead, in turn, to shorter handsoff time ${ }^{24} \mathrm{We}$ agree with this conclusion and propose more concise prompts such as "begin CPR" together with short messages indicating, for instance, the elapsed time every 30 seconds and at the end of the two minutes of CPR, according to the current guidelines.

As per the CPR guide assisting devices only two AEDs possess such feature. Even in this case the judgment is quite personal and objective considerations cannot be made. The potential usefulness of these devices aimed to increase the quality of chest compression is recognized. Studies by Smart and Skorning and their relative coworkers on simulated scenarios of cardiac arrest in manikin feedback devices highlighted the effects of a better CPR quality during chest compression $[27,28]$. However, when applied in the clinical setting, there is yet no evidence that the use of these devices is associated with an increased rate of return of spontaneous circulation and of hospital discharge [29].

Our study has some evident limitation part of whose were already addressed in the previous publication [15]. All tests were made on a bench where the engineering devices cannot simulate the real clinical conditions. Part of Savastano's study provides data of the AEDs in the real setting [27]. Aside the bench tests, our group is planning to analyze the AED traces from the real cardiac arrest scenarios.

As a second issue, the data on the time intervals until shock/no shock indication (Table 2) in some AED were missing and the tests could not be repeated as those devices were no longer available. Thirdly, we did not test the CPR assisting devices in terms of efficacy but rather we just assessed whether they were present or not on the AEDs. Lastly, we recognize that newer models emerged on the market. However, the AEDs herein investigated are currently used in many hospitals, EMS Services and in public facilities. Nevertheless, our study provides additional support to the already existing literature in this field as it is the only study that took into consideration a higher number of AEDs by also examining parameters not previously investigated. Finally, it adds data that are consistent with the other reports thus providing an overall comprehension of these devices from the bench to the simulated manikin scenarios to the real setting. As already stated by Savastano and co-workers no major improvements have been made since the earlier investigations on their characteristics more than a decade ago [26]. Further research on the AEDs is needed to identify areas of improvement to increase survival following cardiac arrest.

\section{Conclusion}

This study is the conclusive part of previous investigations on commercially available AEDs conducted at the AREU's Engineering facility. As new devices are available on the market, the AED manufacturing Companies should take the advantage of these data. Within the several features that distinguish each device, some characteristics are of paramount importance such as those that determine a too long hands-off time intervals and these need to be improved.

\section{Conflict of interest}

The authors state the absence of conflict of interest at the time of study design, data collection and manuscript preparation. Some of the AED models described were subsequently purchased at regional level and by several private Emergency Associations linked to the Regional Emergency Trust without affecting the results and the conclusions of the present manuscript.

\section{References}

1. Marenco JP, Wang PJ, Link MS, Homoud MK, Estes NA (2001) Improving survival from sudden cardiac arrest: the role of the automated external defibrillator. JAMA 285 : 1193-2000

2. Kitamura T, Iwami T, Kawamura T, Nagao K, Tanaka H, et al. (2010) Nationwide public-access defibrillation in Japan. N Engl J Med 362: 994-1004. [Crossref]

3. Weisfeldt ML, Sitlani CM, Ornato JP, Rea T, Aufderheide TP, et al. (2010) Surviva after application of automatic external defibrillators before arrival of the emergency medical system: evaluation in the resuscitation outcomes consortium population of 21 million. J Am Coll Cardiol 55: 1713-1720. [Crossref]

4. Agerskov M, Nielsen AM, Hansen CM, Hansen MB, Lippert FK, et al. (2015) Public Access Defibrillation: Great benefit and potential but infrequently used. Resuscitation 96: 53-58. [Crossref]

5. Blom MT, Beesems SG, Homma PC, Zijlstra JA, Hulleman M et al. (2014) Improved survival after out-of-hospital cardiac arrest and use of automated external defibrillators. Circulation 130: 1868-1875.

6. Berdowski J, Blom MT, Bardai A, Tan HL, Tijssen JG, et al. (2011) Impact of onsite or dispatched automated external defibrillator use on survival after out-of-hospital cardiac arrest. Circulation 124: 2225-2232. [Crossref]

7. Ringh M, Rosenqvist M, Hollenberg J, Jonsson M, Fredman D, et al. (2015) Mobilephone dispatch of laypersons for CPR in out-of-hospital cardiac arrest. $N$ Engl J Med 372: 2316-2325. [Crossref]

8. Kramer-Johansen J, Edelson DP, Abella BS, Becker LB, Wik L, et al. (2007) Pauses in chest compression and inappropriate shocks: a comparison of manual and semiautomatic defibrillation attempts. Resuscitation 73: 212-220. [Crossref]

9. Edelson DP, Abella BS, Kramer-Johansen J, Wik L, Myklebust H, et al. (2006) Effects of compression depth and pre-shock pauses predict defibrillation failure during cardiac arrest. Resuscitation 71:137-45. [Crossref]

10. Cheskes S, Schmicker RH, Verbeek PR, David DS, Siobhan PB, et al (2014) Resuscitation Outcomes Consortium (ROC) investigators. The impact of peri-shock pause on survival from out-of-hospital shockable cardiac arrest during the Resuscitation Outcomes Consortium PRIMED trial. Resuscitation 85: 336-342. [Crossref]

11. Cheskes S, Schmicker RH, Christenson J, et al. (2011) Resuscitation Outcomes Consortium (ROC) Investigators. Peri-shock pause: an independent predictor of survival from out-of-hospital shockable cardiac arrest. Circulation 124: 58-66.

12. Christenson J, Andrusiek D, Everson-Stewart S, et al. (2009) Resuscitation Outcome Consortium Investigators. Chest compression fraction determines survival in patients with out-of-hospital ventricular fibrillation. Circulation 120: 1241-1247.

13. Perkins GD, Handley AJ, Koster RW, Castrén M, Smyth MA et al. (2015) European Resuscitation Council Guidelines for Resuscitation 2015. Section 2. Adult basic life support and automated external defibrillation. Resuscitation 95: 81-99. [Crossref] 
14. Locatelli A, Kette F, Bozzola M, Zoli A (2012) A comparison of different AEDs features: Lighting, analysis time, CRP prompts. Resuscitation 83: e57.

15. Kette F, Locatelli A, Bozzola M, Zoli A, Li Y, et al. (2013) Electrical features of eighteen Automated External Defibrillators: A systematic evaluation. Resuscitation 84: 1596-603. [Crossref]

16. Kette F, Locatelli A, Bozzola M, Zoli A (2014) What is ventricular tachycardia for Automated External Defibrillators? J Clin Exper Cardiol 5: 1.

17. Ristagno G, Tang W, Russell JK, Jorgenson D, Wang H, et al. (2008) Minimal interruption of cardiopulmonary resuscitation for a single shock as mandated by automated external defibrillations does not compromise outcomes in a porcine model of cardiac arrest and resuscitation. Crit Care Med 36: 3048-3053. [Crossref]

18. Sun S, Weng Y, Wu X, Tang K, Ye S, et al. (2011) Optimizing the duration of CPR prior to defibrillation improves the outcome of CPR in a rat model of prolonged cardiac arrest. Resuscitation 82 Supp1 2: S3-S7. [Crossref]

19. Meaney PA, Bobrow BJ, Mancini ME, et al. (2013) CPR Quality Summit Investigators, the American Heart Association Emergency Cardiovascular Care Committee, and the Council on Cardiopulmonary, Critical Care, Perioperative and Resuscitation. Cardiopulmonary resuscitation quality: improving cardiac resuscitation outcomes both inside and outside the hospital: a consensus statement from the American Heart Association. Circulation 128: 417-435.

20. Olasveengen TM, Wik L, Kramer-Johansen J, Sunde K, Pytte M, et al. (2007) Is CPR quality improving? A retrospective study of out-of-hospital cardiac arrest. Resuscitation 75: $260-266$.

21. Achleitner U, Amann A, Stoffaneller M, Baubin M (1999) Waveforms of external defibrillators: analysis and energy contribution. Resuscitation 41: 193-200.
22. Achleitner U, Rheinberger K, Furtner B, Amann A, Baubin M (2001) Waveform analysis of biphasic external defibrillators. Resuscitation 50: 61-70.

23. Snyder D, Morgan C (2004) Wide variation in cardiopulmonary resuscitation interruption intervals among commercially available automated external defibrillators may affect survival despite high defibrillation efficacy. Crit Care Med 32 (Suppl.) S421-S424.

24. Rhee JE, Kim T, Kim K, Choi S (2009) Is there any room for shortening hands-off time further when using an AED? Resuscitation 80: 231-237. [Crossref]

25. Berg MD, Clark LL, Valenzuela TD, Kern KB, Berg RA (2005) Post-shock ches compression delays with automated external defibrillator use. Resuscitation 64: 287-291.

26. Savastano S, Vanni V, Burkart R, Raimondi M, Canevari F, et al. (2017) Comparative performance assessment of commercially available automatic external defibrillators: A simulation and real-life measurement study of hands-off time. Resuscitation 110 12-17. [Crossref]

27. Smart JR, Kranz K, Carmona F, Lindner TW, Newton A (2015) Does real time objective feedback competition improve performance and quality in manikin CPR training - A prospective observational study from several European EMS. Scand J Trauma Resusc Emerg Med 23: 79.

28. Skorning M, Derwall M, Brokmann JC, Rortgen D, Bergrath S, et al. (2011) Externa chest compressions using a mechanical feedback device: cross-over simulation study. Anaesthesist 60: 717-722. [Crossref]

29. Kirkbright S, Finn J, Tohira H, Bremner A, Jacobs I, et al. (2014) Audiovisual feedback device use by health care professionals during CPR: a systematic review and metaanalysis of randomised and non-randomised trials. Resuscitation 85: 460-471.

Copyright: $\odot 2018$ Kette F. This is an open-access article distributed under the terms of the Creative Commons Attribution License, which permits unrestricted use, distribution, and reproduction in any medium, provided the original author and source are credited. 